Supplement of Atmos. Meas. Tech., 13, 4589-4600, 2020

https://doi.org/10.5194/amt-13-4589-2020-supplement

(C) Author(s) 2020. This work is distributed under

the Creative Commons Attribution 4.0 License.

(c) (1)
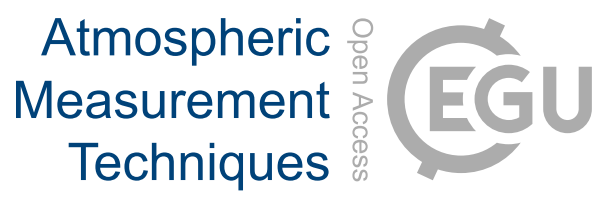

Supplement of

\title{
Characteristics and performance of wind profiles as observed by the radar wind profiler network of China
}

\section{Boming Liu et al.}

Correspondence to: Jianping Guo (jpguocams@gmail.com)

The copyright of individual parts of the supplement might differ from the CC BY 4.0 License. 
Table S1. Detail information of Chinese radar wind profiler stations.

\begin{tabular}{|c|c|c|c|c|c|}
\hline$I D$ & Latitude $\left({ }^{\circ}\right)$ & Longitude $\left({ }^{\circ}\right)$ & Altitude (m) & $\begin{array}{c}\text { Type of } \\
\text { profiler }\end{array}$ & $\begin{array}{c}\text { Land cover } \\
\text { types" }\end{array}$ \\
\hline 51463 & 44 & 88 & 935 & $\mathrm{LC}$ & 13 \\
\hline 52754 & 37 & 100 & 3301 & LC & 0 \\
\hline 52889 & 36 & 104 & 1517 & LC & 13 \\
\hline 53588 & 39 & 114 & 2208 & LC & 10 \\
\hline 54338 & 41 & 122 & 5 & PB & 13 \\
\hline 54342 & 42 & 124 & 50 & LC & 13 \\
\hline 54399 & 40 & 116 & 46 & LC & 13 \\
\hline 54406 & 40 & 116 & 489 & LC & 12 \\
\hline 54419 & 40 & 117 & 75 & LC & 10 \\
\hline 54421 & 41 & 117 & 286 & LC & 10 \\
\hline 54424 & 40 & 117 & 32 & LC & 12 \\
\hline 54511 & 40 & 116 & 32 & LC & 13 \\
\hline 54525 & 40 & 117 & 5 & LC & 13 \\
\hline 54527 & 39 & 117 & 5 & LC & 13 \\
\hline 54597 & 40 & 116 & 409 & LC & 9 \\
\hline 54619 & 39 & 117 & -3 & LC & 12 \\
\hline 54727 & 37 & 118 & 121 & LC & 13 \\
\hline 54736 & 37 & 119 & 11 & LC & 13 \\
\hline 54752 & 38 & 121 & 60 & LC & 12 \\
\hline 54843 & 37 & 119 & 22 & LC & 13 \\
\hline 54857 & 36 & 120 & 12 & PB & 13 \\
\hline 56462 & 29 & 102 & 2925 & LC & 1 \\
\hline 57171 & 34 & 113 & 142 & LC & 12 \\
\hline 57358 & 31 & 111 & 295 & LC & 8 \\
\hline 57377 & 31 & 112 & 184 & LC & 13 \\
\hline 57494 & 31 & 114 & 23 & LC & 13 \\
\hline 57516 & 30 & 106 & 230 & LC & 13 \\
\hline 57633 & 29 & 109 & 795 & LC & 8 \\
\hline 57687 & 28 & 113 & 119 & LC & 9 \\
\hline 57793 & 28 & 114 & 130 & LC & 9 \\
\hline 57989 & 25 & 114 & 105 & PA & 2 \\
\hline 57996 & 25 & 114 & 148 & LC & 9 \\
\hline 58026 & 34 & 118 & 25 & LC & 12 \\
\hline 58044 & 35 & 119 & 9 & LC & 12 \\
\hline 58135 & 33 & 118 & 17 & LC & 13 \\
\hline 58141 & 34 & 119 & 19 & LC & 12 \\
\hline 58158 & 33 & 120 & 5 & LC & 12 \\
\hline 58235 & 32 & 119 & 19 & LC & 13 \\
\hline 58237 & 32 & 119 & 49 & LC & 9 \\
\hline 58238 & 32 & 119 & 41 & LC & 13 \\
\hline 58246 & 33 & 120 & 15 & LC & 13 \\
\hline 58259 & 32 & 121 & 5 & LC & 12 \\
\hline 58334 & 31 & 118 & 8 & LC & 9 \\
\hline 58339 & 31 & 119 & 25 & LC & 13 \\
\hline 58340 & 32 & 119 & 30 & LC & 9 \\
\hline 58352 & 32 & 121 & 20 & LC & 0 \\
\hline
\end{tabular}




\begin{tabular}{|c|c|c|c|c|c|}
\hline 58353 & 32 & 121 & 17 & LC & 13 \\
\hline 58354 & 32 & 120 & 15 & LC & 13 \\
\hline 58356 & 31 & 121 & 20 & LC & 13 \\
\hline 58358 & 31 & 120 & 11 & LC & 9 \\
\hline 58365 & 31 & 121 & 16 & LC & 13 \\
\hline 58367 & 31 & 121 & 4 & LC & 13 \\
\hline 58377 & 31 & 121 & 30 & LC & 13 \\
\hline 58448 & 30 & 120 & 112 & LC & 8 \\
\hline 58450 & 31 & 120 & 4 & LC & 9 \\
\hline 58452 & 31 & 121 & 4 & LC & 13 \\
\hline 58459 & 30 & 120 & 38 & LC & 13 \\
\hline 58460 & 31 & 121 & 6 & LC & 13 \\
\hline 58462 & 31 & 121 & 0 & LC & 12 \\
\hline 58463 & 31 & 122 & 8 & LC & 9 \\
\hline 58474 & 31 & 122 & 49 & LC & 0 \\
\hline 58527 & 29 & 117 & 88 & LC & 9 \\
\hline 58543 & 30 & 119 & 175 & LC & 0 \\
\hline 58557 & 29 & 120 & 100 & LC & 13 \\
\hline 58637 & 28 & 118 & 150 & LC & 13 \\
\hline 58730 & 28 & 118 & 224 & LC & 9 \\
\hline 58737 & 27 & 118 & 154 & LC & 2 \\
\hline 58754 & 27 & 120 & 36 & LC & 8 \\
\hline 58760 & 28 & 121 & 71 & LC & 0 \\
\hline 58821 & 27 & 117 & 286 & LC & 2 \\
\hline 58822 & 27 & 117 & 342 & LC & 8 \\
\hline 58845 & 27 & 120 & 87 & LC & 5 \\
\hline 58912 & 26 & 117 & 404 & LC & 12 \\
\hline 58917 & 25 & 116 & 307 & LC & 9 \\
\hline 58921 & 26 & 117 & 253 & LC & 8 \\
\hline 58927 & 25 & 117 & 375 & LC & 9 \\
\hline 58933 & 27 & 119 & 869 & LC & 8 \\
\hline 58935 & 26 & 118 & 640 & LC & 9 \\
\hline 58938 & 25 & 119 & 65 & LC & 13 \\
\hline 58942 & 26 & 119 & 51 & LC & 14 \\
\hline 58944 & 26 & 120 & 18 & LC & 13 \\
\hline 59046 & 24 & 109 & 312 & LC & 13 \\
\hline 59072 & 25 & 112 & 131 & LC & 9 \\
\hline 59125 & 24 & 117 & 108 & LC & 9 \\
\hline 59137 & 25 & 119 & 124 & LC & 13 \\
\hline 59140 & 25 & 118 & 20 & LC & 0 \\
\hline 59268 & 23 & 112 & 70 & PA & 9 \\
\hline 59284 & 23 & 113 & 42 & LC & 13 \\
\hline 59285 & 24 & 114 & 39 & LC & 9 \\
\hline 59287 & 23 & 113 & 65 & PA & 9 \\
\hline 59290 & 24 & 114 & 79 & LC & 9 \\
\hline 59294 & 23 & 114 & 30 & LC & 2 \\
\hline 59303 & 24 & 116 & 133 & LC & 9 \\
\hline 59312 & 24 & 117 & 59 & LC & 13 \\
\hline 59320 & 24 & 117 & 20 & LC & 14 \\
\hline 59431 & 23 & 109 & 101 & LC & 9 \\
\hline 59462 & 23 & 112 & 53 & LC & 9 \\
\hline
\end{tabular}




\begin{tabular}{cccccc}
59476 & 23 & 113 & 37 & LC & 13 \\
59486 & 23 & 114 & 75 & LC & 13 \\
59488 & 22 & 113 & 30 & LC & 0 \\
59758 & 20 & 110 & 65 & LC & 9 \\
59948 & 18 & 110 & 368 & LC & 2 \\
59981 & 17 & 112 & 20 & LC & 0 \\
G7190 & 22 & 112 & 119 & LC & 0 \\
M7100 & 32 & 118 & 5 & LC & 12 \\
\hline
\end{tabular}

* The type mark $P A, P B$, and $L C$ represent tropospheric type I , tropospheric type I, and boundary layer radar wind profiler.

* The land cover types 0-16 represent the water, Evergreen Needleleaf forest, Evergreen Broadleaf forest, Deciduous Needleleaf forest, Deciduous Broadleaf forest, Mixed forest, Closed shrublands, Open shrublands, woody savannas, Savannas, Grasslands, Permanent Croplands, Urban and built-up, Cropland/Natural vegetation mosaic, Snow and ice, Barren or sparsely vegetated, respectively. 